\title{
Characterization of PLZT Ceramics for Optical Sensor and Actuator Devices
}

\author{
Ribal Georges Sabat \\ Royal Military College of Canada \\ Canada
}

\section{Introduction}

Perovskite Lead Lanthanum Zirconate Titanate (PLZT) ceramics have the following chemical formula $\mathrm{Pb}_{1-x} \mathrm{La}_{x}\left(\mathrm{Zr}_{y}, \mathrm{Ti}_{1-y}\right)_{1-0.25 x} \mathrm{~V}^{\mathrm{B}} \mathrm{B.25x}_{\mathrm{x}} \mathrm{O}_{3}$ and are typically known as PLZT $(100 x / 100 y / 100(1-y))$. Compositional changes within this quaternary ferroelectric system, especially along the morphotropic phase boundaries, can significantly alter the material's properties and behaviour under applied electric fields or temperature variations. This allows such a system to be tailored to a variety of transducer applications. For instance, PLZT ceramics have been suggested for use in optical devices (Glebov et al. 2007; Liberts, Bulanovs, and Ivanovs 2006; Wei et al. 2011; Ye et al. 2007; Zhang et al. 2009) because of their good transparency from the visible to the near-infrared, and their high refractive index ( $n \approx 2.5$ ), which is advantageous in light wave guiding applications (Kawaguchi et al. 1984; Thapliya, Okano, and Nakamura 2003). PLZT compositions near the tetragonal and rhombohedral ferroelectric phases and anti-ferroelectric/cubic phases, typically with compositions $(a / 65 / 35)$ with $7<a<12$, are known as relaxor ferroelectrics, since they exhibit a frequency-dependent diffuse ferroelectric-paraelectric phase transition in their complex dielectric permittivity. Relaxor ferroelectrics are particularly attractive in transducer applications because they can be electrically or thermally induced into a ferroelectric phase possessing a large dipole moment accompanied by a large mechanical strain, and revert back to a non-ferroelectric state upon the removal of the field or temperature. They also exhibit a slim hysteretic behaviour in the transition region, upon the application of an electric field, making them ideal for precise control actuator applications.

In this chapter, I will conduct a review on some of the fundamental material properties of relaxor ferroelectric PLZT ceramics, which include the dielectric, ferroelectric, electromechanical, electro-optical and thermo-optical behaviours. Further details on each section can be found in the references (Lévesque and Sabat 2011; Sabat, Rochon, and Mukherjee 2008; Sabat and Rochon 2009b; Sabat and Rochon 2009c; Sabat and Rochon 2009a).

\section{Dielectric properties}

The temperature and frequency dependence of the dielectric properties of any ferroelectric material are essential features to study, since they provide insight to possible transducer 
characteristics, such as the electrostrictive and electro-optic effects, which are both consequences of dipole moments arising from ion displacements.

Transparent PLZT (9.5/65/35) ceramics, having a thickness of $0.64 \mathrm{~mm}$, were cut in $10 \mathrm{~mm}$ squares. Forty-nanometer layers of gold were sputtered on opposing faces and conducting wires were glued to each surface to act as electrodes. The complex dielectric permittivity was measured using an impedance analyzer at a frequency range from 0.12 to $5000 \mathrm{kHz}$. The temperature at which the measurements were taken could be varied since the samples were placed inside a thermal chamber. The probing ac electric field of the impedance analyzer was set at amplitude of $1 \mathrm{~V}$ and the heating rate was approximately $1{ }^{\circ} \mathrm{C} / \mathrm{min}$, starting at $-60^{\circ} \mathrm{C}$ up to $100^{\circ} \mathrm{C}$. The relative permittivity and loss tangent can be respectively calculated from the real and imaginary parts of the dielectric permittivity.

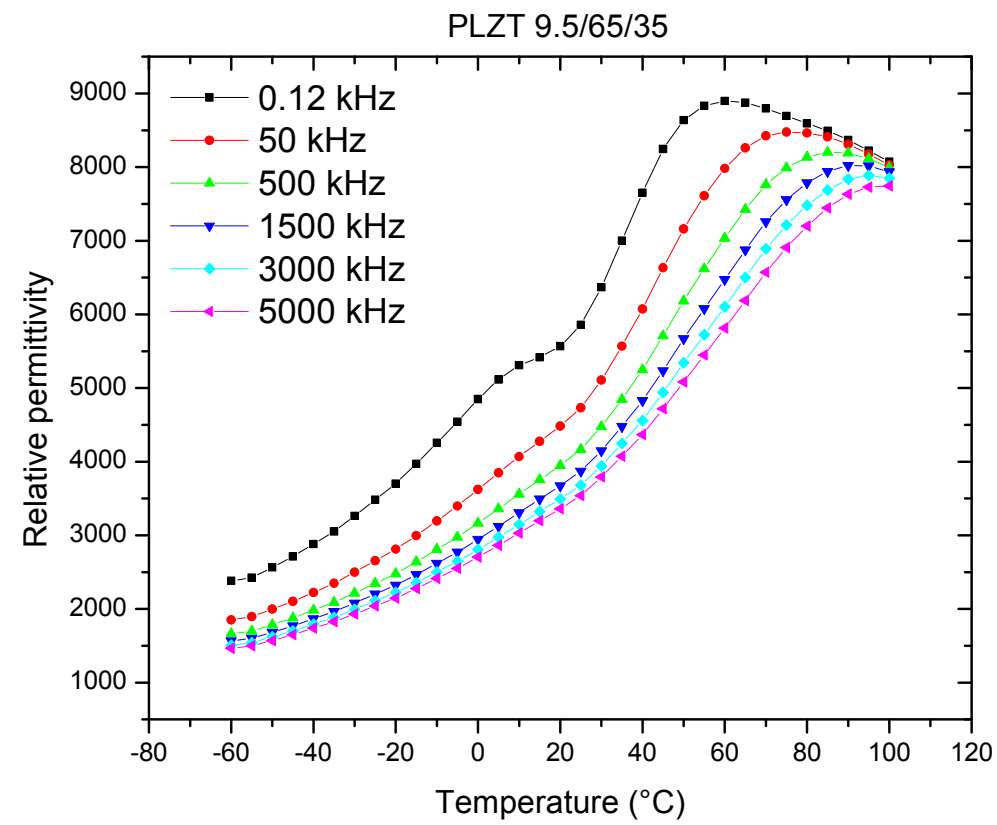

Fig. 1. Real relative permittivity of PLZT $(9.5 / 65 / 35)$ as a function of temperature and frequency.

Figures 1 and 2 respectively show the temperature dependence of the relative permittivity and loss tangent of relaxor ferroelectric PLZT (9.5/65/35). As the temperature increases from $-60^{\circ} \mathrm{C}$ to $100^{\circ} \mathrm{C}$, the relative permittivity generally increased due to the unfreezing of domains. Between $0^{\circ} \mathrm{C}$ and $10^{\circ} \mathrm{C}$, a broad peak can be seen in the lower frequency curves. This peak corresponds to the diffuse phase transition in this relaxor ceramic from the ferroelectric to the paraelectric state (also called the relaxor phase). Further heating continued to increase the relative dielectric permittivity until a maximum was achieved, at which point, the crystal's structure became cubic. This maximum in the permittivity, which is frequency dependent, occurs at the Curie temperature. Evidence of these phase transitions can also be seen in the loss tangent graph in figure 2 . 


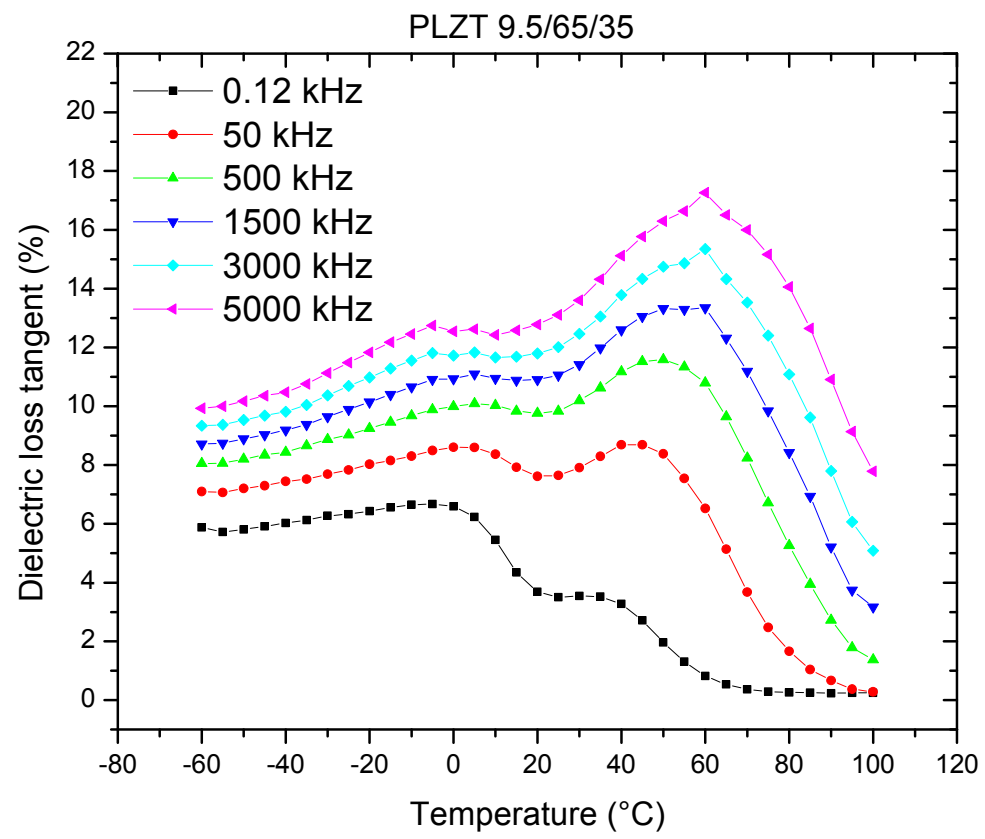

Fig. 2. Loss tangent of PLZT as a function of temperature and frequency.

The relative permittivity in figure 1 seems to decrease with increasing frequency, while the loss tangent in figure 2 increases at higher frequencies. These two observations go hand-inhand since the frequency response of the complex permittivity is highly affected by the ability of ferroelectric domains and dipoles to rotate with the applied electric field. At higher frequencies, the ceramic material is no longer able to store as much electric energy in the dipoles and the relative permittivity decreases. As a consequence, a larger portion of the input energy is transferred to heating the ceramic and the loss tangent increases.

\section{Ferroelectric properties}

A Sawyer-Tower circuit (Sawyer and Tower 1930), with a $9.8 \mu \mathrm{F}$ series capacitance, was used to measure the ferroelectric hysteresis at room temperature. Figures 3 and 4 respectively show the electric displacement of PLZT (9.5/65/35) and PLZT (9.0/65/35) ceramics as a function of a dc bias electric field. The field was first increased from zero to +1.7 MV.m ${ }^{-1}$, back down to $-1.7 \mathrm{MV} . \mathrm{m}^{-1}$, and finally up to zero. This cycle lasted 50 seconds and was repeated 3 consecutive times. Typical relaxor ferroelectric hysteretic curves were observed for these two compositions.

Figures 3 and 4 clearly illustrate how such a small change in the chemical composition of the PLZT can strongly affect the material's properties: PLZT (9.5/65/35) samples appear to possess a higher electric displacement compared to PLZT $(9.0 / 65 / 35)$ at identical field values, but the hysteresis is slightly slimmer for the $(9.0 / 65 / 35)$ composition samples. From Haertling's room temperature phase diagram of PLZT (Haertling 1987), it can be seen that 
the relaxor ferroelectric compositions studied here are located near the intersection of several other crystal phases including the ferroelectric-tetragonal, ferroelectricrhombohedral and the antiferroelectric phase. Remnants of antiferroelectric hystereses can be found in both figures, but it's more evident for PLZT (9.5/65/35).

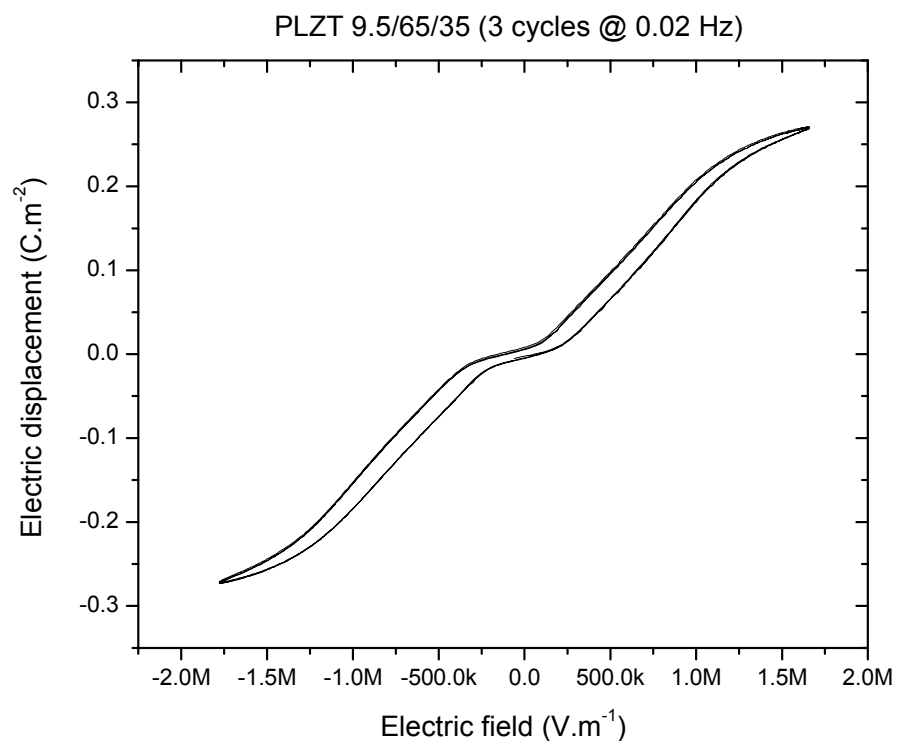

Fig. 3. Electric displacement of PLZT (9.5/65/35) as a function of dc electric fields.

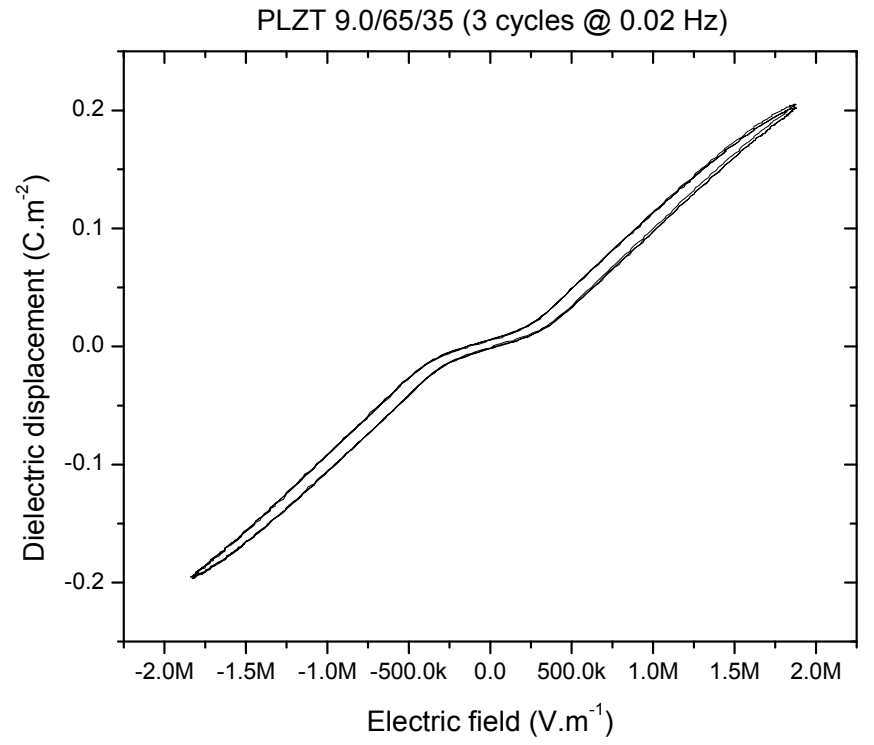

Fig. 4. Electric displacement of PLZT (9.0/65/35) as a function of dc electric fields. 
According to the dielectric results in the previous section, decreasing the temperature at which these measurements were taken should increase the hysteresis gap (ferroelectric behaviour), and increasing the temperature should further decrease the hysteresis. This was observed by Carl et al. (Carl and Geisen 1973) for PLZT (9.0/65/35). The slim ferroelectric behaviour of these PLZT compositions makes them ideal for use as precision sensors and actuators, since they have almost no remnant polarization when the field is removed. Hence, the risk of depoling is eliminated in this case.

\section{Electrostrictive and piezoelectric properties}

\subsection{Theory}

Electrostriction refers to the elastic deformation of all dielectric materials upon the application of an electric field. Unlike piezoelectricity, the electrostrictive strain is quadratic to the electric field and reversal of the field doesn't reverse the strain direction. The basic phenomenology of electrostriction in materials is discussed in detail in many texts (Lines and Glass 1977; Jona and Shirane 1962; Mason 1958; Mason 1950). For a dielectric material under isothermal, adiabatic and stress-free conditions, upon the application of an electric field $E_{k}$, the strain tensor $S_{i j}$ can be written as:

$$
S_{i j}=\sum_{1}^{3} d_{i j k} E_{k}+\sum_{1}^{3} \gamma_{i j k l} E_{k} E_{l}+\ldots
$$

Where $d_{i j k}$ is the piezoelectric coefficient and $\gamma_{i j k l}$ is the electrostriction coefficient. For the case where the applied electric field is in the 3-direction, which is taken to be the direction perpendicular to a sample's electrodes, equation (1) becomes:

$$
S_{3}=d_{33} E_{3}+\gamma_{333} E_{3}^{2}+\ldots
$$

If all the coefficients of equation (2) are known, one can accurately predict the longitudinal strain under a varying electric field for a given piezoelectric or electrostrictive material, and even for a material exhibiting both piezoelectric and electrostrictive effects, such as irreversible electrostrictive materials. For ideal reversible electrostrictive materials, which possess no remnant polarization at zero electric field, the odd power term of the electric field in equation (2) vanishes. However, we will consider the relaxor PLZT ceramics studied in this chapter as irreversible electrostrictives, to account for any ferroelectric behaviour under dc bias fields, and we will therefore include both terms of the electric field in equation (2).

If a sinusoidal electric field with a dc bias component $E_{3}=E_{D C}+E_{0} \cos (\omega t)$ is applied to an irreversible electrostrictive material, such as relaxor PLZT, equation (2) becomes:

$$
S_{3}=d_{33}\left(E_{D C}+E_{0} \cos (\omega t)\right)+\gamma_{333}\left(E_{D C}+E_{0} \cos (\omega t)\right)^{2}+\ldots
$$

Which can be re-arranged as follows:

$$
S_{3}=\ldots+\left[d_{33} E_{0}+2 \gamma_{333} E_{D C} E_{0}+\ldots\right] \cos (\omega t)+\left[1 / 2 \gamma_{333} E_{0}^{2}+\ldots\right] \cos (2 \omega t)+\ldots
$$


Hence, first, second and other harmonics should be present in the strain response of the PLZT ceramic. Experimentally, peaks should appear in the Fourier-transformed strain measurements at one and two times the driving frequency. By fitting the frequency-domain experimental strain peaks to the corresponding term in equation (4), the strain-electric-field longitudinal material coefficients $\left(d_{33}\right.$ and $\left.\gamma_{333}\right)$ can be obtained.

\subsection{Experiment}

To measure the strain developed by the PLZT ceramics upon the application of an electric field, a Doppler laser vibrometer can be used. The vibrometer uses the heterodyne technique to acquire the characteristic motion of the vibrating samples, as opposed to the homodyne method which uses the electronic equivalent of the fringe counting principle to measure strain. The vibrometer's helium-neon laser beam was pointed at one of the gold-coated surfaces of a vibrating PLZT sample and scattered back from it; the opposing sample surface was glued onto a fixed copper plate. The velocity and amplitude of the vibrating sample surface creates a phase or frequency modulation of the scattered laser light due to the Doppler effect. Using suitable decoders, the sample velocity information can be recovered from the frequency modulation of the Doppler signal, while the displacement information can be reconstructed from the phase modulation available at the same time.

If the velocity decoder on the vibrometer is used, the displacement information can still be obtained by integrating the velocity signal as a function of time. Fourier analysis can be performed to transform the displacement-amplitude-versus-time signal into the frequency domain. Even though the laser vibrometer can achieve picometer resolution, it can't detect strain by a dc voltage alone. Only alternating compressive and expansive strain can be measured with this method. Fortunately, this includes combined ac and dc field excitations.

Since the relaxor PLZT compositions used in this study have a cubic crystal structure at room temperature, the expectation is that the strain generated should be mostly quadratic, especially if no dc bias is applied. Figure 5 shows the Fourier-transformed displacement amplitude as a function of frequency for a PLZT (9.5/65/35) ceramic under a combination of both ac and dc bias electric fields. As expected, several frequency peaks are seen; this indicates that the material is vibrating at harmonic frequencies to the applied field.

Figure 6 shows the ac strain amplitude versus ac field amplitude, measured up to the fourth harmonic, of PLZT (9.5/65/35) without any dc bias. The second harmonic electrostrictive strain is pre-dominant. It rapidly increased with the ac field and reached a maximum of $0.00026 \mathrm{~m} \cdot \mathrm{m}^{-1}$ at $0.5 \mathrm{MV} \cdot \mathrm{m}^{-1}$. The theoretical curve seen in this figure is the result of fitting the data collected at $240 \mathrm{~Hz}$ to the second harmonic term in equation (4).

Figure 7 shows the ac strain amplitude versus dc bias fields, measured up to the fourth harmonic, with a driving 0.37 MV.m-1 peak-to-peak ac field at $120 \mathrm{~Hz}$ for PLZT (9.5/65/35). In this case, the first harmonic piezoelectric strain is dominant and seems to increase with the dc bias field until a maximum is reached at $1.2 \mathrm{MV} \cdot \mathrm{m}^{-1} \mathrm{dc}$. The theoretical curve seen in this figure is the result of fitting the data collected at $120 \mathrm{~Hz}$ to the first harmonic term in equation (4), while fixing the ac field value. This general behaviour of relaxor ferroelectrics has been previously observed for PMN electrostrictive ceramics (Masys et al. 2003). 


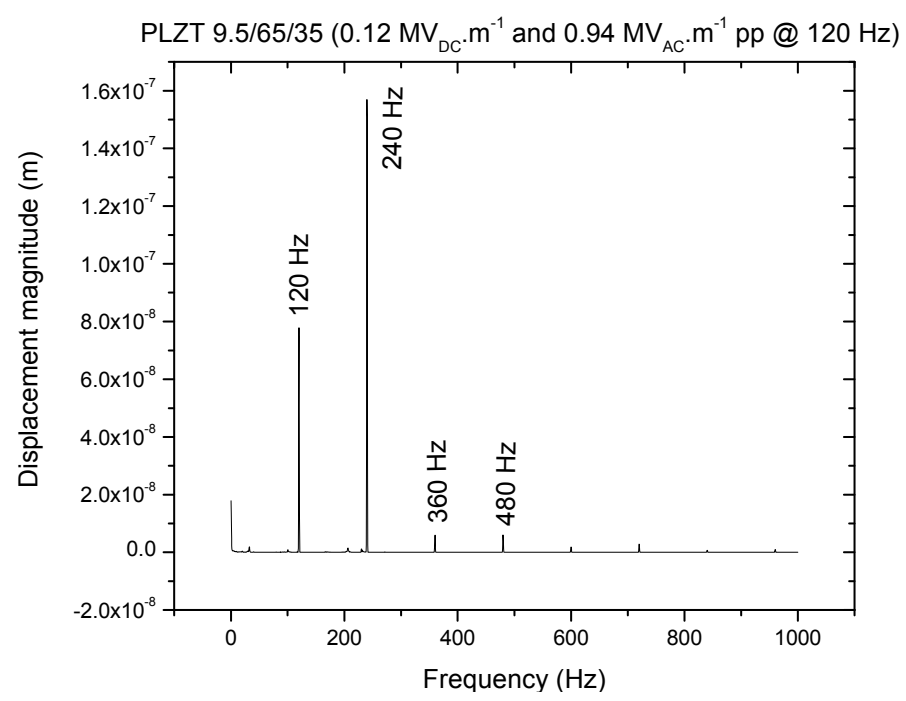

Fig. 5. Displacement magnitude of PLZT (9.5/65/35) as a function of frequency.

The increased strain with increasing dc bias in figure 7 can be explained by previous dielectric measurements of PLZT (9.0/65/35) ceramics as a function of both temperature and dc bias (Bobnar et al. 1999): They have observed a sharp increase in the dielectric permittivity with increasing dc bias fields at temperatures close to the ferroelectric-relaxor phase transition, indicating that the dc bias is inducing the creation of electric dipoles at this transition, and hence increasing the overall piezoelectric response.

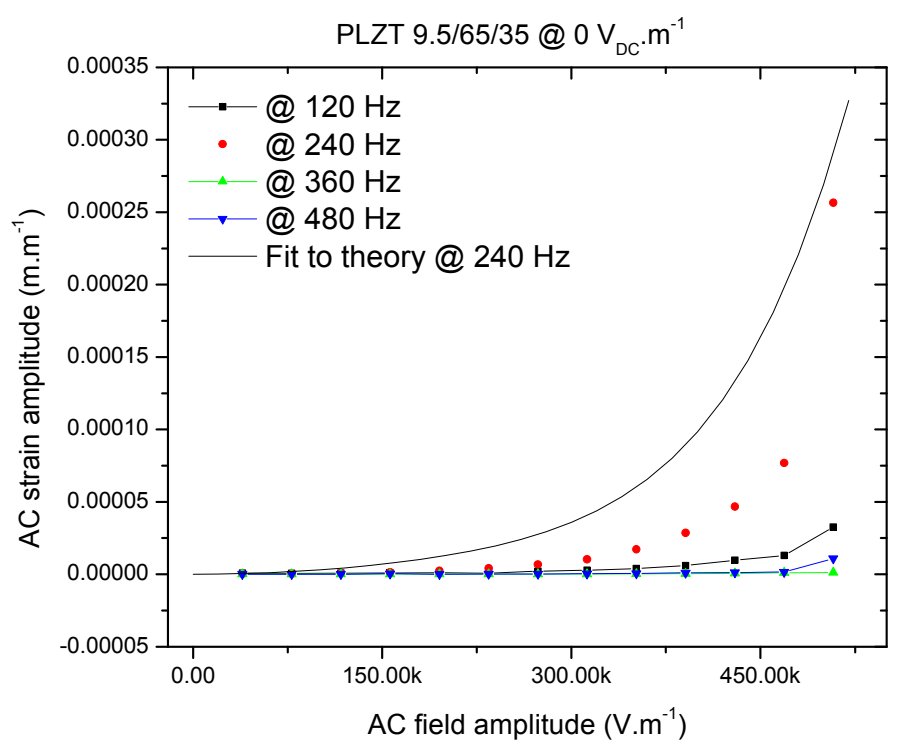

Fig. 6. Strain amplitude of PLZT $(9.5 / 65 / 35)$ as a function of ac electric field. 


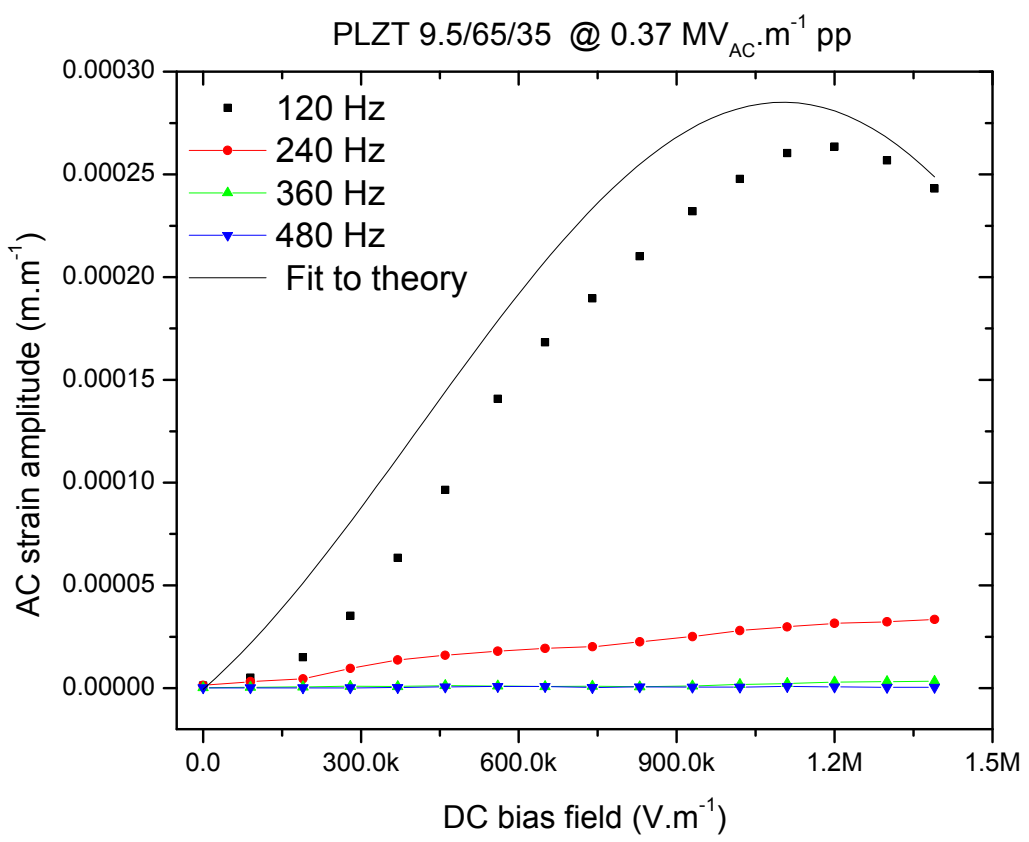

Fig. 7. Strain amplitude of PLZT (9.5/65/35) as a function of dc bias electric field.

This behaviour could also be explained as a consequence of the dc bias field induced reorientation of the polar nano-regions, favouring their alignment in the direction of the field (Tagantsev and Glazounov 1999). It is equally possible that, since the sample's composition is located near the ferroelectric tetragonal/rhombohedral boundary on the PLZT phase diagram, a dc bias induced phase transition from paraelectric to ferroelectric would increase the number of available polarization states, thus, maximizing the strain.

Next, the ac strain amplitude was plotted as a function of dc bias fields for various driving ac fields, as seen in figure 8 . The first-harmonic piezoelectric strain increased with both the ac and dc fields until a maximum of approximately $0.8 \times 10^{-3} \mathrm{~m} \cdot \mathrm{m}^{-1}$ occurred at $1.1 \mathrm{MV} \cdot \mathrm{m}^{-1}$ $\mathrm{dc}$ and $1.09 \mathrm{MV} \cdot \mathrm{m}^{-1}$ ac peak-to-peak. These results once again confirm studies of the dielectric behaviour of PLZT (9.0/65/35) (Bobnar et al. 1999), in which above a critical field, called $\mathrm{E}_{\mathrm{c}}$, a phase transition from relaxor to ferroelectric occurs in the PLZT structure. It is perhaps the presence of both phases simultaneously that give rise to the piezoelectric strain; further increasing of the fields would just render the samples more and more ferroelectric, therefore decreasing the strain.

The theoretical fitting curves seen in figures 6 to 8 were all fitted simultaneously to the first and second harmonic terms in equation (4), and the longitudinal piezoelectric and electrostrictive material coefficients were determined for PLZT (9.5/65/35) and PLZT $(9.0 / 65 / 35)$, as seen in table 1 . It can be noted that the experimental results and the theory are generally in good agreement. The small discrepancy between theory and experiment can be associated with random experimental uncertainties. 


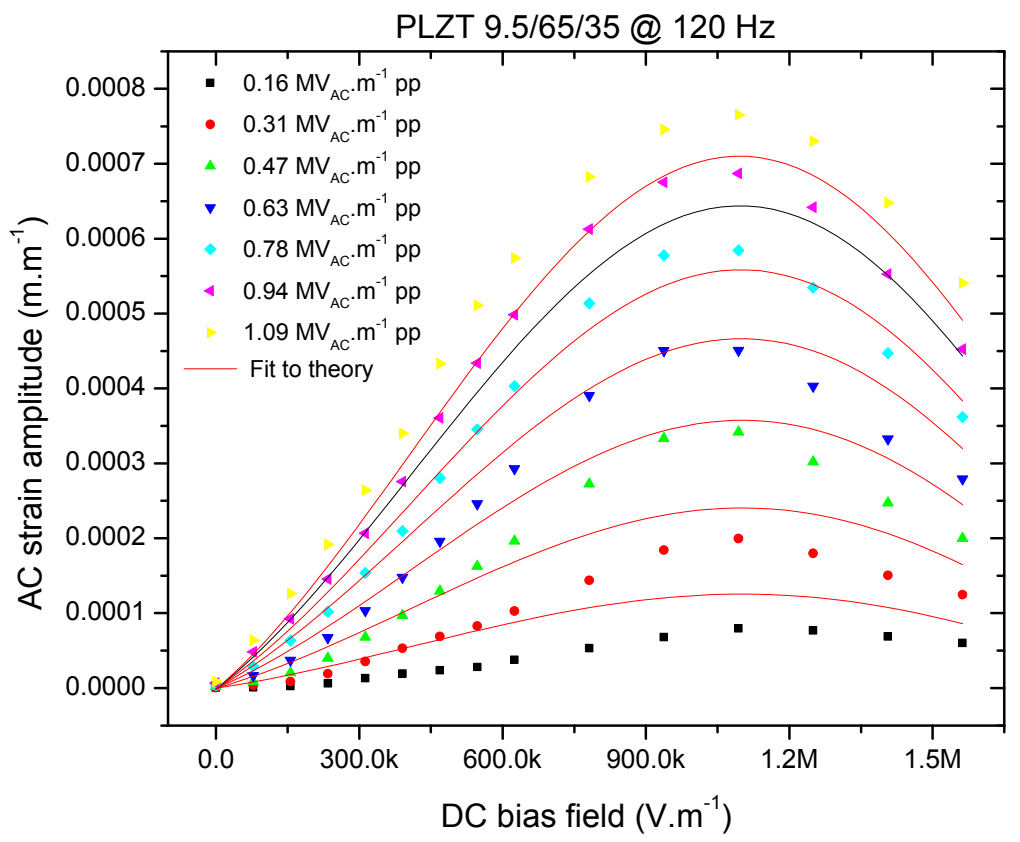

Fig. 8. Strain amplitude of PLZT $(9.5 / 65 / 35)$ as a function of dc bias electric field at various ac field amplitudes.

\begin{tabular}{cccccc}
\hline \multirow{2}{*}{ Material coefficient } & Units & \multicolumn{2}{c}{$(\mathbf{9 . 5 / 6 5 / 3 5 )}$ PLZT } & \multicolumn{2}{c}{$\mathbf{( 9 . 0 / 6 5 / 3 5 )}$ PLZT } \\
& & Value & Error & Value & Error \\
\hline$d_{33}$ & $\left({\left.\mathrm{~m} . \mathrm{V}^{-1}\right) \times 10^{-11}}\right.$ & -0.34 & 2.44 & 5.65 & 4.93 \\
$\gamma_{333}$ & $\left(\mathrm{~m}^{2} \cdot \mathrm{V}^{-2}\right) \times 10^{-16}$ & 6.14 & 0.64 & 3.18 & 0.83 \\
\hline
\end{tabular}

Table 1. Strain material coefficients of PLZT.

\section{Electro-optic and thermo-optical properties}

\subsection{Fabry-Pérot method}

Many previously published papers have reported on the dc field electro-optic properties of PLZT ceramics (Haertling and Land 1971; Haertling 1971; Fogel, BarChaim, and Seidman 1980; Goldring et al. 2003). In this section, the electro-optic effects of large driving ac fields and the superposition of ac and dc electric fields are studied on relaxor PLZT ceramics using an interferometric technique. Two-hundred-nanometers thick gold electrodes were sputtered on both sides of each PLZT sample while leaving $\sim 3 \mathrm{~mm}$ gap in the centre of each face, as illustrated in figure 9. 


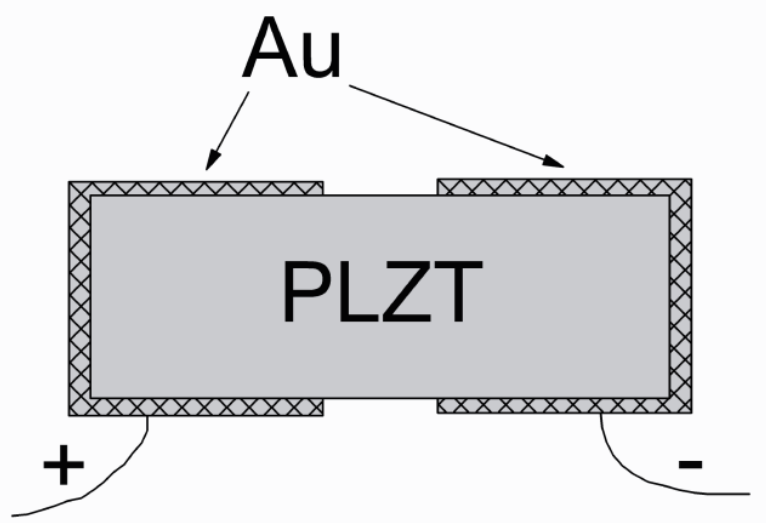

Fig. 9. Top view representation of the gold-sputtered electrodes on a PLZT sample.

The transmission of a laser light beam through the sample will exhibit a Fabry-Pérot interference pattern as the light incidence angle changes (Hecht 1987). Upon the application of any electric field, the angular interference fringes will shift due to a change in the optical path length within the sample. Depending on the input light's polarization, the individual refractive index variations, denoted $\Delta n_{1}$ and $\Delta n_{3}$, can be measured individually. The 3subscript indicates the direction of the applied field, while the 1-subscript is the direction orthogonal to it. Figure 10 shows an example of a Fabry-Pérot interference pattern.

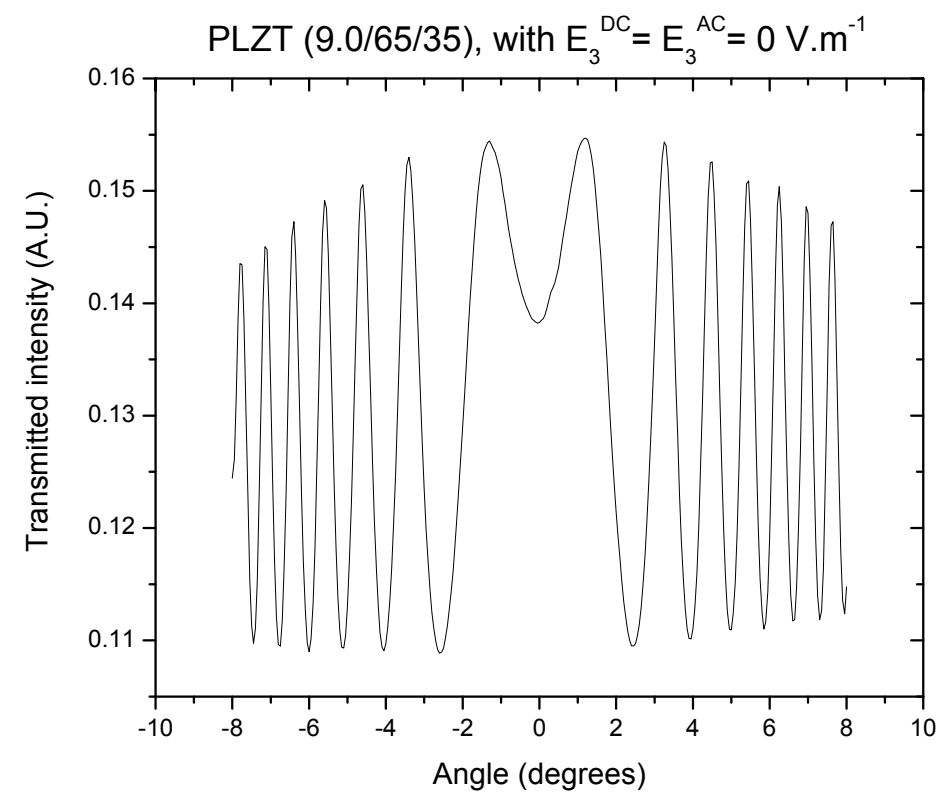

Fig. 10. Fabry-Pérot interference on a PLZT (9.0/65/35) sample with no applied fields. 
The condition for the intensity maxima could be written as:

$$
(n d)^{2}-d^{2} \sin ^{2} \theta=\left(\frac{m \lambda}{2}\right)^{2}, \quad m=0,1,2 \ldots
$$

where $n$ is the refractive index of the PLZT, $d$ its thickness, $\theta$ is the angle of incidence of the laser and $\lambda$ is the wavelength of the laser. Assuming a change in both the refractive index and the thickness of the PLZT under an electric field, one would obtain from equation (5) the change in the optical path length between a zero field and an applied field condition:

$$
\Delta(n d)=\frac{d}{2 n}\left(\sin ^{2} \theta_{2}-\sin ^{2} \theta_{1}\right)
$$

Where $\theta_{1}$ is the incidence angle corresponding to an intensity maximum at a zero field condition and $\theta_{2}$ is the same intensity maximum angle under an arbitrary applied field $E_{3}$. But, since $\Delta(n d)=n \Delta d+d \Delta n$, further development would lead to:

$$
\Delta n=\frac{\left(\sin ^{2} \theta_{2}-\sin ^{2} \theta_{1}\right)}{2 n}-S_{3} n
$$

Where $S_{3}$ is the longitudinal strain due to the arbitrary transverse electric field. It was previously found that the ratio of $\Delta n / S$ for unclamped relaxor PLZT ceramics was approximately 16 (Carl and Geisen 1973), therefore, the second term in equation (7) should equate to approximately $0.16 \Delta n$, since relaxor PLZT compositions have an approximate refractive index of 2.5 at room temperature. Under mechanical clamping conditions, as is the case illustrated in figure 9, the sample would have restricted movement and hence the second term on the right hand side of equation (7) would become even smaller.

Assuming an increase in the temperature of the tested samples under applied ac fields possibly due to the rapid rotation of domains, thermal expansion of the PLZT ceramic can be contributing to the strain. Therefore, equation (7) can be modified as follows:

$$
\Delta n=\frac{\left(\sin ^{2} \theta_{2}-\sin ^{2} \theta_{1}\right)}{2 n}-S_{3} n-n \alpha \Delta T
$$

Where $\alpha$ is the linear thermal expansion coefficient and $\Delta T$ is the temperature variation. Haertling calculated that the average linear thermal expansion coefficient of various relaxor PLZT ceramics was around $3.9 \times 10^{-6}{ }^{\circ} \mathrm{C}^{-1}$ (Haertling 1971). Therefore, the third term on the right hand side of equation (8) would contribute approximately $10^{-5} \Delta T$ to the refractive index change $\Delta n$ due to thermal expansion. Since there is an experimental error of approximately $\pm 5 \times 10^{-5}$ attributed to the refractive index change found using this method, a hypothetical increase in the temperature of the sample by just a few degrees would have a negligible effect on $\Delta n$. Therefore, the bulk of the change in the optical path length due to an applied electric field and possible thermal expansion should mostly be related to the refractive index change alone. Equation (8) can be approximated as: 


$$
\Delta n \approx \frac{\left(\sin ^{2} \theta_{2}-\sin ^{2} \theta_{1}\right)}{2 n}
$$

\subsection{Room temperature results}

The first set of measurements was conducted on the effect of a dc electric field $E_{3}^{D C}$ on the change in the refractive indices $\Delta n_{3}$ (same direction as the applied electric field) and $\Delta n_{1}$ (perpendicular to the applied electric field and the plane of incidence). In order to measure $\Delta n_{3}$, two polarizers, placed before and after the sample, were set to allow only the Transverse Magnetic (TM) component of the laser light to propagate; the TM component of light have their $\vec{E}$-vector parallel to the applied electric field on the sample. On the other hand, $\Delta n_{1}$ was measured by having both polarizers allow only the Transverse Electric (TE) component of the laser light to pass through to the light detector; the TE component have their $\vec{E}$-vector perpendicular to the applied electric field.

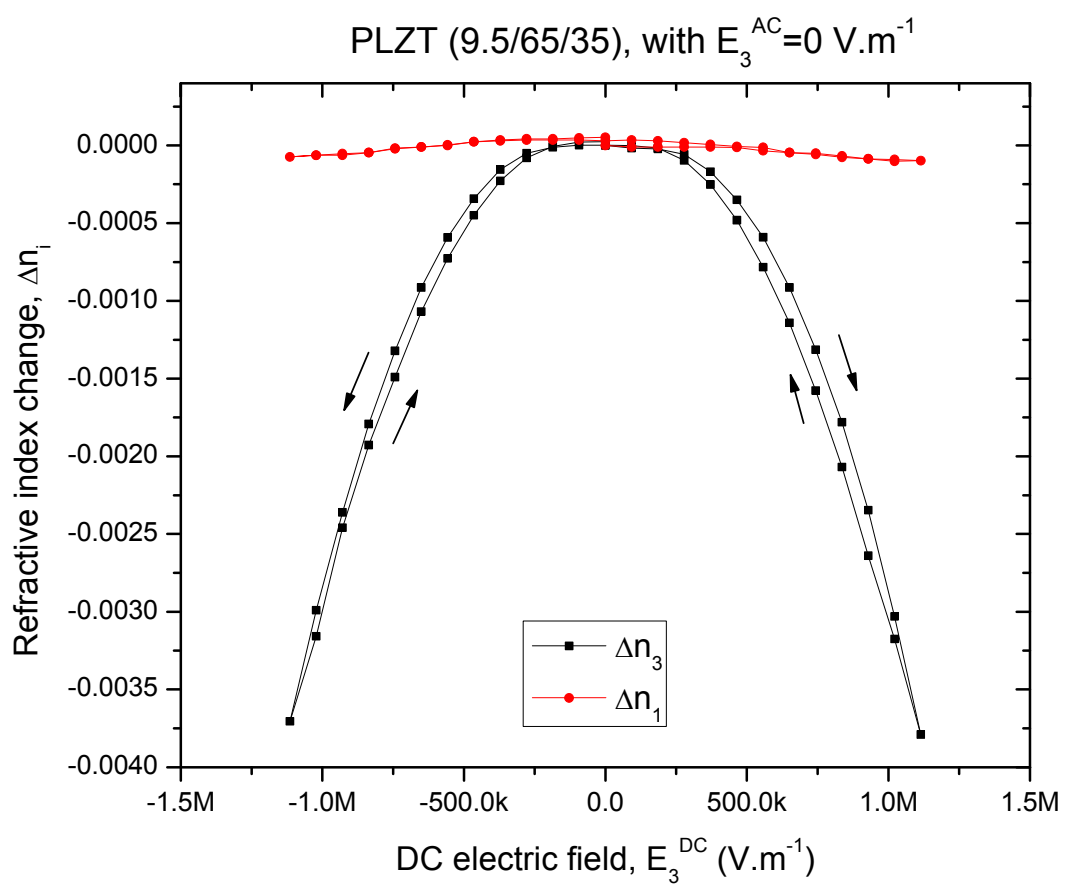

Fig. 11. Refractive index change $\Delta n_{i}$ as a function of dc electric fields $E_{3}^{D C}$ for PLZT $(9.5 / 65 / 35)$.

Figure 11 shows the change in the refractive indices $\Delta n_{1}$ and $\Delta n_{3}$ as a function of an applied dc electric field $E_{3}^{D C}$ for a PLZT $(9.5 / 65 / 35)$ ceramic. The dc field was cycled from 0 to +1.1 MV.m-1 down to $-1.1 \mathrm{MV} . \mathrm{m}^{-1}$, and back up to $0 . \Delta n_{1}$ remained near zero while $\Delta n_{3}$ decreased quadratically with the dc field, due to the electro-optic Kerr effect, until a minimum of - 
0.0037 was reached. It was expected that $\Delta n_{3}$ be much larger than $\Delta n_{1}$ since the dc field favours the creation and alignment of domains along its direction, and hence causing a greater change in the refractive index along its direction. Only a minor hysteresis is observed in the electro-optic behaviour; this mirrors the dielectric results discussed previously.

The temperature of the PLZT ceramic was monitored as an ac electric field, with $1 \mathrm{kHz}$ frequency, was applied. A significant increase of up to $25^{\circ} \mathrm{C}$ was measured for PLZT $(9.5 / 65 / 35)$ at $E_{3}^{A C}=620 \mathrm{kV} \cdot \mathrm{m}^{-1}$. This elevation in the samples' temperature can be attributed to the increased movement of domains trying to align with the alternating field direction and would cause a thermal expansion of the sample. The maximum thermal strain generated should decrease the refractive index change $\Delta n_{i}$ of a tested sample by a maximum of $2.5 \times 10^{-4}$. According to the dielectric measurements, such a temperature increase would also affect the crystal structure of the PLZT by moving it away from the paraelectricferroelectric transition region, around room temperature, towards the paraelectric or relaxor phase.

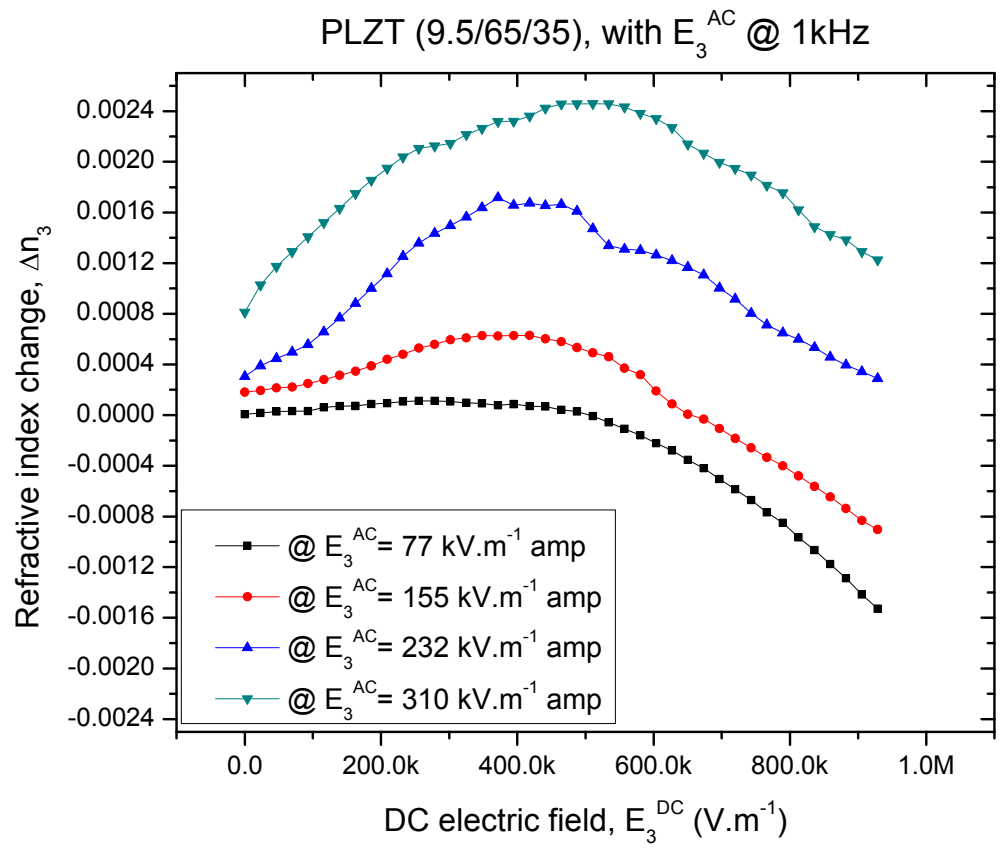

Fig. 12. Refractive index change $\Delta n_{3}$ as a function of dc electric fields $E_{3}^{D C}$ at various ac field amplitudes $E_{3}^{A C} @ 1 \mathrm{kHz}$ frequency for PLZT (9.5/65/35).

Figure 12 shows the refractive index change $\Delta n_{3}$ as a function of a dc electric field $E_{3}^{D C}$ in superposition with various values of an ac field $E_{3}^{A C}$ at $1 \mathrm{kHz}$ frequency. The effect of increasing ac fields were found to be opposite to increasing dc fields for PLZT (9.5/65/35) ceramics; $\Delta n_{3}$ decreased with the dc field, but increased with the ac field. A broad peak can 
be seen in this figure around $E_{3}^{D C}=0.5 \mathrm{MV} \cdot \mathrm{m}^{-1}$ and this peak becomes more pronounced as the ac field amplitude increased. These results hint to the presence of a critical threshold dc field $E_{c}$, below which, increasing ac fields cause the destruction, misalignment and rapid rotation of domains, exhibited by an increase in the overall temperature of the sample and the subsequent transition to the relaxor phase, and above which, increasing dc bias fields favour the stabilization of the ferroelectric phase and promotes the presence of electric dipoles and their alignment along the dc field direction.

\subsection{Temperature effects on the electro-optic properties}

The same experimental set-up, as described above, was used, except the samples were now placed in a temperature-controlled chamber. As seen in figure 13, the temperature of a PLZT $(9.5 / 65 / 35)$ was varied from $20^{\circ} \mathrm{C}$ down to $-30^{\circ} \mathrm{C}$, up to $60^{\circ} \mathrm{C}$ and down again to $20^{\circ} \mathrm{C}$. Only a slight hysteresis was found for these results. The temperature dependence of the refractive index change $\Delta n$ of PLZT (9.5/65/35), without any applied field, was found to be linear in the above range, with a positive slope of $\Delta n / \Delta T=(8.57 \pm 0.25) \times 10^{-5}{ }^{\circ} \mathrm{C}^{-1}$. The third term in equation (8), which corresponds to the linear thermal expansion, contributed approximately $11 \%$ to the calculated slope.

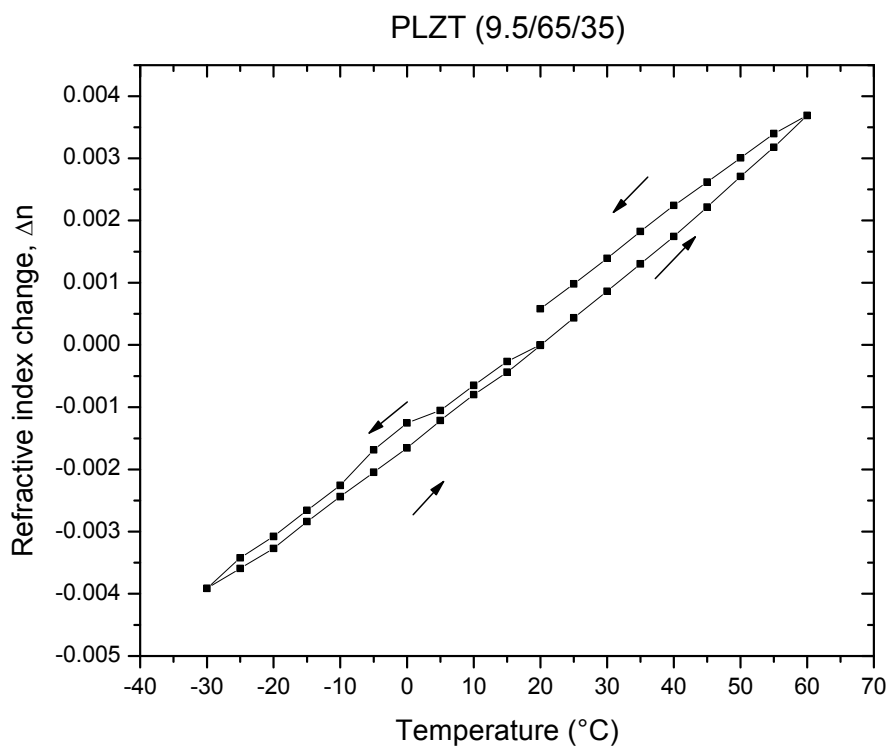

Fig. 13. Temperature dependence of the refractive index $\Delta n$ of PLZT (9.5/65/35).

The temperature coefficient of the refractive index of this tested PLZT composition is in good agreement with the value of $(7.1 \pm 0.3) \times 10^{-5}{ }^{\circ} \mathrm{C}^{-1}$ previously obtained using the thermal-lens method (Falcão et al. 2006).

An increase in a ceramic's temperature would increase its specific volume, thus decreasing its density, and consequently, its refractive index. But, according to Prod'homme 
(Prod'homme 1960), it also increases the electronic polarizability of the sample due to a decrease in size of the atomic groupings responsible for the polarization, as the structure tends to a more dissociated state, which in turn causes the refractive index to increase gradually. These two contradicting effects can be explained by the results of figure 13, and equation (8): the linear thermal expansion decreased $\Delta n$ as the temperature increased, but the shift in the Fabry-Pérot pattern clearly indicates a much stronger increase of $\Delta n$ with increasing temperature. Therefore, the electronic polarizability had a greater impact on the temperature dependence of the refractive index of the PLZT ceramic compared to the thermal expansion.

We have seen that relaxor ferroelectric PLZT ceramics, with compositions (a/65/35) with $7<\mathrm{a}<12$, undergo a thermally or electrically induced diffuse and frequency-dependent paraelectric (or relaxor) to long-range ferroelectric phase transition. PLZT (9.5/65/35) ceramics undergo this phase change around $5{ }^{\circ} \mathrm{C}$. However, no evidence of this phase change is seen in figure 13. This is likely because the specific refractivity of materials $R$, which is a measure of the electronic polarization, is unaffected by this particular phase transition. This refractivity constant is defined by the Lorentz-Lorenz relation:

$$
R=\frac{n^{2}-1}{n^{2}+2} \cdot V
$$

Where $\mathrm{n}$ is the refractive index and V is the specific volume. Differentiating equation (10) with respect to temperature yields (Prod'homme 1960):

$$
\frac{\Delta n}{\Delta T}=\frac{\left(n^{2}-1\right)\left(n^{2}+2\right)}{6 n}(\varphi-\beta)
$$

Since the first term of the right hand side of equation (11) can be considered a constant, the temperature coefficient of the refractive index $\Delta n / \Delta T$ is a measure of the difference between the electronic polarization coefficient $\phi$ and the volumetric thermal expansion coefficient $\beta$, where $\phi$ and $\beta$ are defined as:

$$
\begin{gathered}
\varphi=\frac{1}{R} \frac{d R}{d T} \\
\beta=\frac{1}{V} \frac{d V}{d T} \cong 3 \cdot \alpha
\end{gathered}
$$

Therefore, with the results of figure 13, the polarization coefficient $\phi$ for PLZT $(9.5 / 65 / 35)$ is $(4.14 \pm 0.09) \times 10^{-5}{ }^{\circ} \mathrm{C}^{-1}$, by using an approximate value of $\beta \cong 3 \cdot \alpha=1.2 \times 10^{-5}{ }^{\circ} \mathrm{C}^{-1}$.

Figures 14 and 15 show the temperature dependence of $\Delta n_{3}$ and $\Delta n_{1}$ under a dc bias field $E_{3}^{D C}=774 \mathrm{kV} \cdot \mathrm{m}^{-1}$ for PLZT $(9.5 / 65 / 35)$. The application of a dc bias, at a given temperature, decreased $\Delta n_{3}$ while $\Delta n_{1}$ remained unchanged. It was found that $\Delta n_{3} / \Delta T$ increased over $\Delta n / \Delta T$, but $\Delta n_{1} / \Delta T$ remained somewhat unchanged. This indicates that the dc bias only increased the electronic polarizability of the test sample along the 3-axis, the direction of the applied field. All the temperature coefficients of the refractive indices for PLZT (9.5/65/35) 
and PLZT (9.0/65/35) were obtained and are summarized in table 2. Since the signs of all the calculated $\Delta n / \Delta T$ are positive, this means that the polarization coefficient $\phi$ of these materials is increasing at a larger rate than the volumetric thermal expansion $\beta$ with increasing temperature.

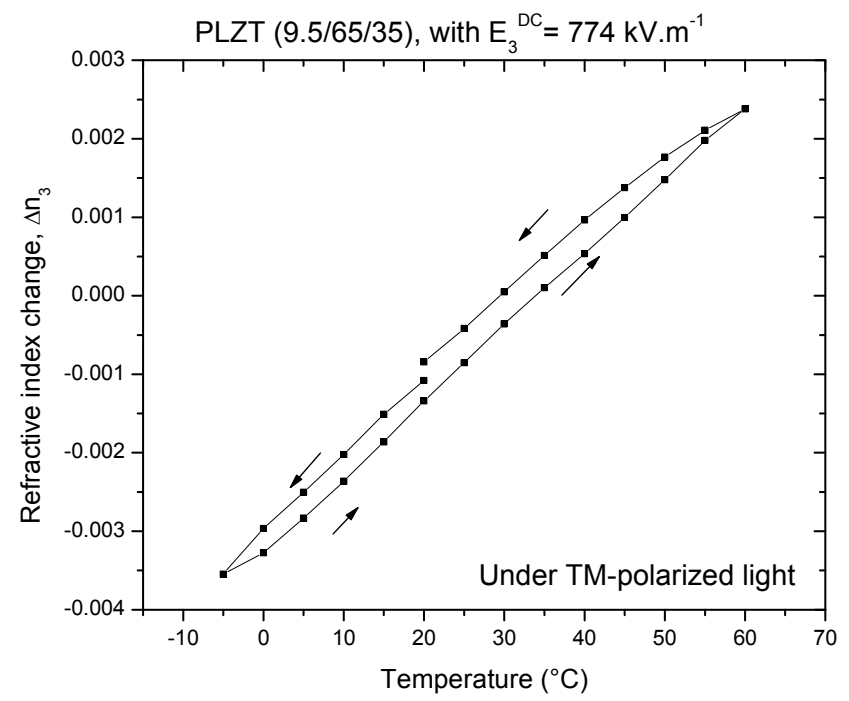

Fig. 14. Temperature dependence of the refractive index $\Delta n_{3}$ of PLZT $(9.5 / 65 / 35)$ under dc bias.

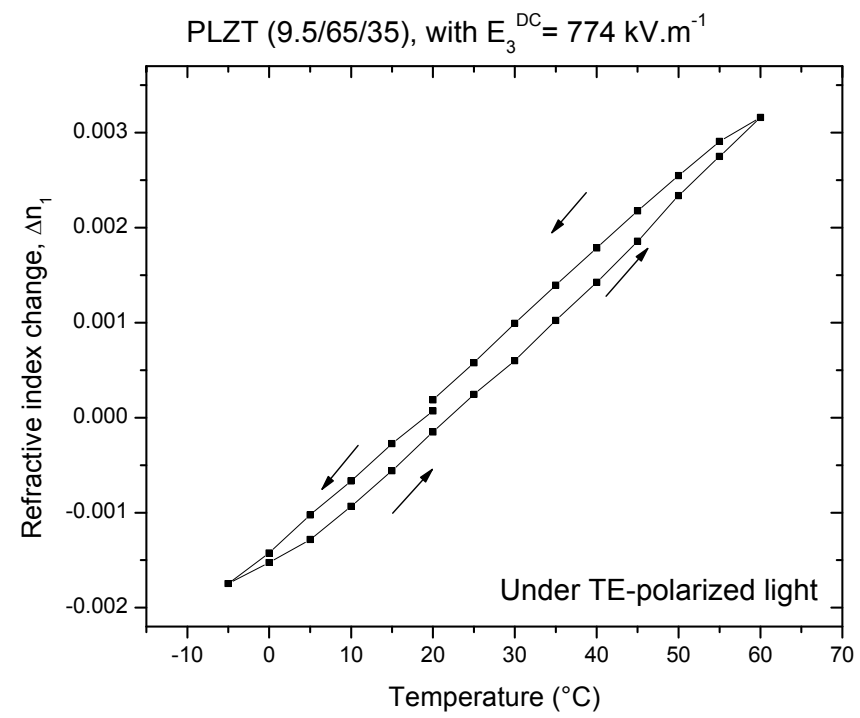

Fig. 15. Temperature dependence of the refractive index $\Delta n_{1}$ of PLZT $(9.5 / 65 / 35)$ under dc bias. 


\begin{tabular}{cccccc}
\hline $\begin{array}{c}\text { Parameter } \\
\left(\mathbf{\times ~ 1 0 - 5} \mathbf{~}^{-1}\right)\end{array}$ & $\begin{array}{c}E_{3}^{D C} \\
\left(\mathbf{k V . m} \mathbf{m}^{-1}\right)\end{array}$ & \multicolumn{2}{c}{$\mathbf{( 9 . 5 / 6 5 / 3 5 )}$ PLZT } & \multicolumn{2}{c}{$\mathbf{( 9 . 0 / 6 5 / 3 5 )}$ PLZT } \\
\hline$\frac{\Delta n}{\Delta T}$ & 0 & 8.57 & 0.25 & 7.28 & 0.16 \\
$\frac{\Delta n_{3}}{\Delta T}$ & 774 & 9.41 & 0.42 & 8.11 & 0.30 \\
$\frac{\Delta n_{1}}{\Delta T}$ & 774 & 7.85 & 0.32 & 7.47 & 0.26 \\
\hline
\end{tabular}

Table 2. The measured temperature coefficients of the refractive index of relaxor PLZT ceramics.

Figure 16 shows the refractive index change $\Delta n_{3}$ for PLZT $(9.5 / 65 / 35)$ as a function of dc bias fields up to $1 \mathrm{MV} \cdot \mathrm{m}^{-1}$ and at temperatures of 0,20 and $40^{\circ} \mathrm{C}$.

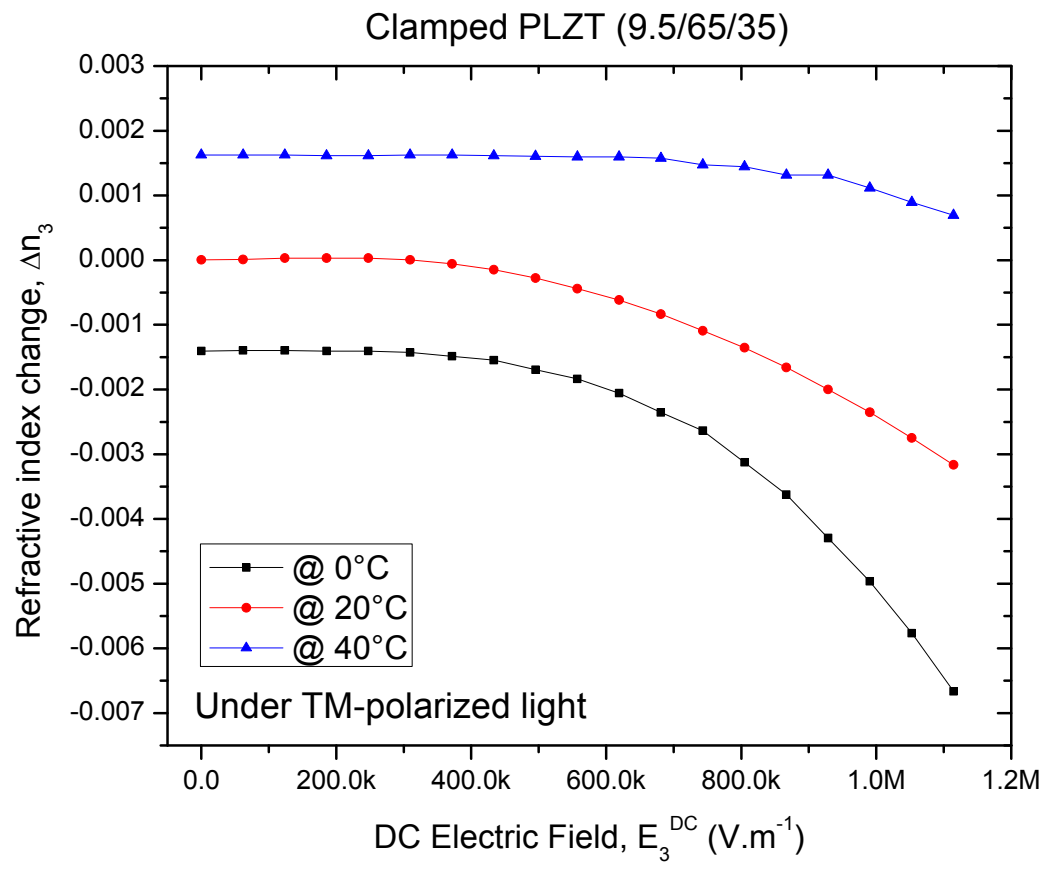

Fig. 16. Dc bias electric field dependence of the refractive index $\Delta n_{3}$ of PLZT (9.5/65/35), at 0,20 and $40^{\circ} \mathrm{C}$. 
The Kerr quadratic electro-optic coefficient $K_{33}$ in the presence of a dc field $E_{3}^{D C}$ can be obtained from (Narasimhamurty 1981):

$$
\Delta n_{3}=-\frac{1}{2} n^{3} K_{33}\left(E_{3}^{D C}\right)^{2}
$$

The various $K_{33}$ coefficients were calculated using equation (14) and reported in table 3. It was found that $K_{33}$ seems to decrease with increasing temperature for both PLZT $(9.5 / 65 / 35)$ and $(9 / 65 / 35)$. This is due to the ferroelectric to relaxor phase change occurring around the tested temperature range.

$$
K_{33} \times 10^{-16}\left(\mathrm{~m}^{2} \cdot V^{-2}\right)
$$

\section{Temperature}

(9.5/65/35) PLZT

(9.0/65/35) PLZT

\begin{tabular}{ccccc} 
& Value & Error & Value & Error \\
\hline $0^{\circ} \mathrm{C}$ & 5.12 & 0.04 & 2.18 & 0.02 \\
$20^{\circ} \mathrm{C}$ & 3.33 & 0.02 & 1.54 & 0.02 \\
$40^{\circ} \mathrm{C}$ & 0.79 & 0.02 & 0.88 & 0.02 \\
\hline
\end{tabular}

Table 3. The quadratic electro-optic coefficient $K_{33}$ of PLZT at various temperatures.

\subsection{Thermal-lensing effects}

Since PLZT absorbs strongly in the far-infrared, a $\mathrm{CO}_{2}$ laser beam $(\lambda=10.6 \mu \mathrm{m})$ can be used to create a Gaussian temperature distribution at the surface of the ceramic. This temperature distribution will consequently alter the localized refractive index of the PLZT, emulating an optical lens. Hence, a low power visible He-Ne laser, travelling along the same path as the $\mathrm{CO}_{2}$ laser, will get focalized after passing through the thermal lens. The focal distance of the created lens is adjustable and will depend on the input power of the $\mathrm{CO}_{2}$ laser. Figure 17 gives a graphical representation of this phenomenon.

A PLZT (9/65/35) ceramic was positioned on glass slide substrate, which was moved by means of a translation stage. It was brought very near the $\mathrm{CO}_{2}$ focal point while a $\mathrm{CCD}$ camera was positioned underneath the glass slide at a distance of $13.5 \mathrm{~cm}$. Figure 18 shows what was observed. As the $\mathrm{CO}_{2}$ laser power was increased, a focusing of the red light occurs. 


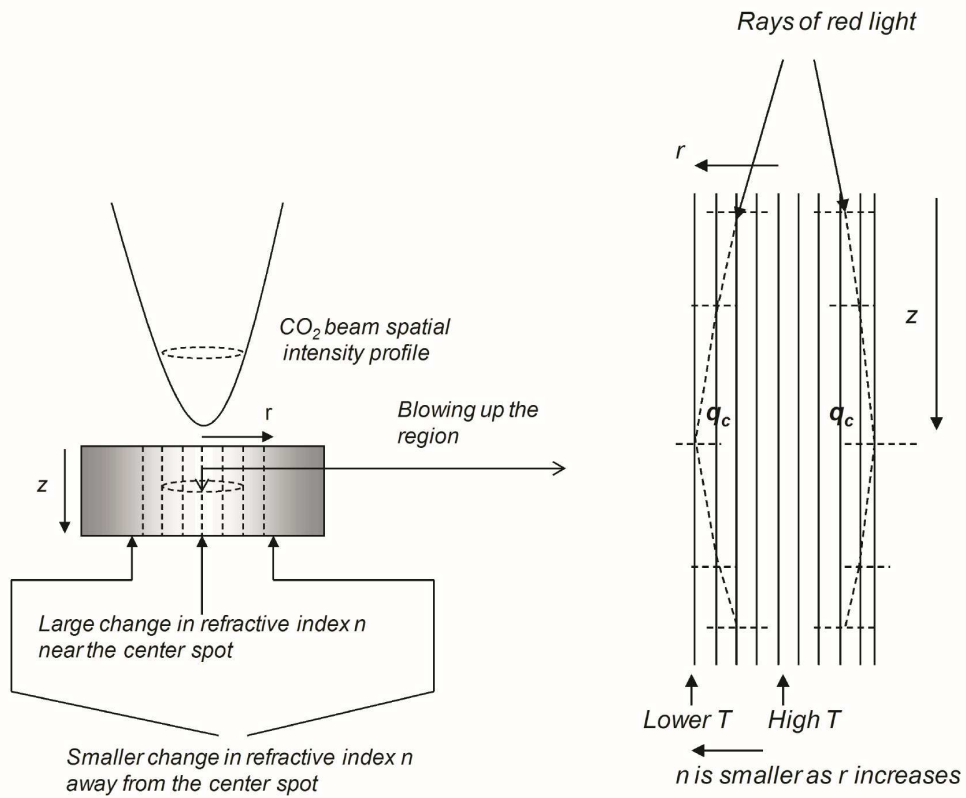

Fig. 17. $\mathrm{CO}_{2}$ beam spatial intensity profile and refractive index gradient near the thick PLZT ceramic surface and self-focusing of a ray of red light resulting from a refractive index gradient near the surface.
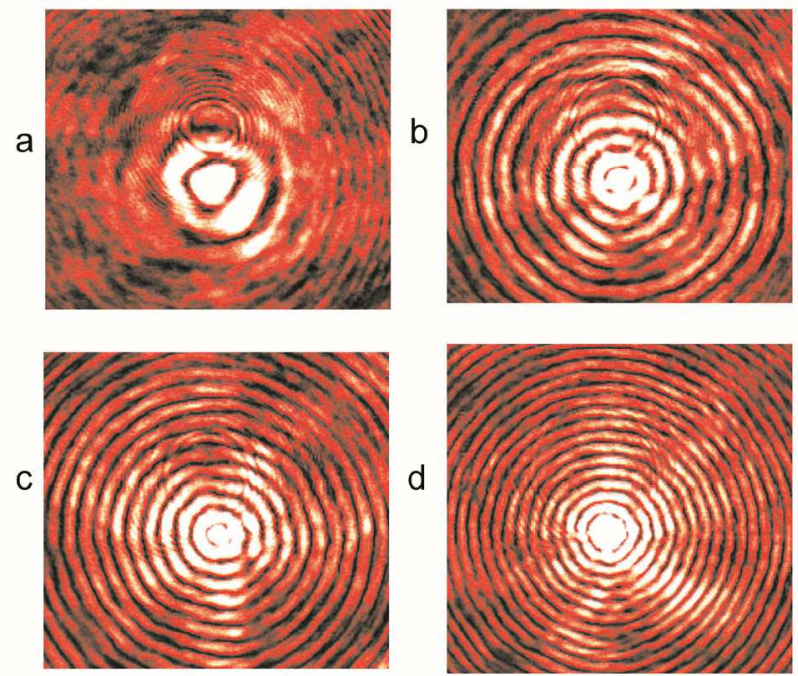

Fig. 18. Thermal lensing for on PLZT (9/65/35). A lens of $35 \mathrm{~mm}$ was used in front of the camera to produce a better view. $\mathrm{CO}^{2}$ laser power a) $\left.2.5 \mathrm{~W} \mathrm{~b}\right) 2.8 \mathrm{~W} \mathrm{c)} 3.2 \mathrm{~W} \mathrm{d)} 3.5 \mathrm{~W}$. 


\section{Conclusion}

Ferroelectrics will also play a major role in the next generation of optical devices. They have the potential to be the backbone of wireless laser communication systems or ground-based fibre optics, capable of carrying large amounts of information at ultrafast speeds. Notwithstanding their industrial and technological uses, ferroelectric materials will also continue to provide consumers with accessories and gadgets that will pleasure and simplify their everyday life. With the recent push on miniaturization of practical devices and the advancement of nanotechnology, ferroelectric materials may be fabricated to exhibit transducer effects at a scale small enough to be useful at the nanometre resolution.

In order to invent and develop future practical applications, we must have a greater fundamental understanding of the properties of these versatile ceramics. This can be accomplished by measuring and characterizing their behaviour under a variety of possible operating conditions. In this chapter, the transducer properties of Lead Lanthanum Zirconate Titanate (PLZT) ceramics were presented. Their dielectric, electromechanical and optical properties have been systematically studied and could provide valuable information for engineers seeking the development or enhancement of practical applications.

\section{References}

Bobnar, V., Kutnjak, Z., Pirc, R., \& Levstik, A. (1999). Electric-field-temperature phase diagram of the relaxor ferroelectric lanthanum-modified lead zirconate titanate. Phys. Rev. B, Vol. 60, No. 9, pp. 6420-6427.

Carl, K., \& Geisen, K. (1973). Dielectric and optical properties of a quasi-ferroelectric PLZT ceramic. Proc. IEEE, Vol. 61, No. 7, pp. 967-974.

Falcão, E. A., Pereira, J. R. D., Santos, I. A., Nunes, A. R., Medina, A. N., Bento, A. C., Baesso, M. L., Garcia, D., \& Eiras, J. A. (2006). Thermo optical properties of transparent PLZT 10/65/35 ceramics. Ferroelectrics, Vol. 336, No. 1, pp. 191.

Fogel, Y., BarChaim, N., \& Seidman, A. (1980). Longitudinal electrooptic effects in slim-loop and linear PLZT ceramics. Appl. Opt., Vol. 19, No. 10, pp. 1609-1617.

Glebov, A. L., Smirnov, V. I., Lee, M. G., Glebov, L. B., Sugama, A., Aoki, S., \& Rotar, V. (2007). Angle selective enhancement of beam deflection in high-speed electrooptic switches. IEEE Photonics Technology Letters, Vol. 19, No. 9, pp. 701.

Goldring, D., Zalevsky, Z., Goldenberg, E., Shemer, A., \& Mendlovic, D. (2003). Optical characteristics of the compound PLZT. Appl. Opt., Vol. 42, No. 32, pp. 6536-6543.

Haertling, G. H. (1987). PLZT electrooptic materials and applications-a review. Ferroelectrics, Vol. 75, pp. 25-55.

__ (1971). Improved hot-pressed electrooptic ceramics in the (pb,la)(zr,ti) $\mathrm{O}_{3}$ system. Journal of the American Ceramic Society, Vol. 54, No. 6, pp. 303-309.

Haertling, G. H., \& Land, C. E. (1971). Hot-pressed (pb,la)(zr,ti) $\mathrm{O}_{3}$ ferroelectric ceramics for electrooptic applications. Journal of the American Ceramic Society, Vol. 54, No. 1, pp. $1-10$.

Hecht, E. (1987). Optics (2nd), Addison-Wesley, .

Jona, F., \& Shirane, G. (1962). Ferroelectric crystalsMacmillan, , New York. 
Kawaguchi, T., Adachi, H., Setsune, K., Yamazaki, O., \& Wasa, K. (1984). PLZT thin-film waveguides. Appl.Opt., Vol. 23, No. 13, pp. 2187-2191.

Lévesque, L., \& Sabat, R. G. (2011). Thermal lensing investigation on bulk ceramics and thinfilm PLZT using visible and far-infrared laser beams. Optical Materials, Vol. 33, No. 3, pp. 460.

Liberts, G., Bulanovs, A., \& Ivanovs, G. (2006). PLZT ceramics based EFISH cell for nonlinear heterodyne interferometry. Ferroelectrics, Vol. 333, No. 1, pp. 81.

Lines, M. E., \& Glass, A. M. (1977). Theory and applications of ferroelectric materialsOxford University Press, , Oxford.

Mason, W. P. (1958). Physical acoustics and the properties of solidsVan Nostrand, , New York.

- - (1950). Piezoelectric crystals and their applications to ultrasonicsVan Nostrand, , New York.

Masys, A. J., Ren, W., Yang, G., \& Mukherjee, B. K. (2003). Piezoelectric strain in lead zirconate titanate ceramics as a function of electric field, frequency, and dc bias. J. Appl. Phys., Vol. 94, No. 2, pp. 1155-1162.

Narasimhamurty, T. S. (1981). Kerr quadratic electro-optic effect: Pockels' phenomenological theory, in: Photoelastic and Electro-Optic Properties of CrystalsAnonymous, pp. 359-362, Plenum Press, , New York.

Prod'homme, L. (1960). Thermal change in the refractive index of glasses. Phys. Chem. Glass., Vol. 1, No. 4, pp. 119-122.

Sabat, R. G., \& Rochon, P. (2009a). The dependence of the refractive index change of clamped relaxor ferroelectric lead lanthanum zirconate titanate (PLZT) ceramics on $\mathrm{AC}$ and DC electric fields measured using an interferometric method. Ferroelectrics, Vol. 386, No. 1, pp. 105.

- - (2009b). Interferometric determination of the temperature dependence of the refractive index of relaxor PLZT ceramics under DC bias. Optical Materials, Vol. 31, No. 10, pp. 1454.

_- - (2009c). Investigation of relaxor PLZT thin films as resonant optical waveguides and the temperature dependence of their refractive index. Appl.Opt., Vol. 48, No. 14, pp. 2649-2654.

Sabat, R. G., Rochon, P., \& Mukherjee, B. K. (2008). Quasistatic dielectric and strain characterization of transparent relaxor ferroelectric lead lanthanum zirconate titanate ceramics. J. Appl. Phys., Vol. 104, No. 5, pp. 054115.

Sawyer, C. B., \& Tower, C. H. (1930). Rochelle salt as a dielectric. Phys.Rev., Vol. 35, No. 3, pp. 269-273.

Tagantsev, A. K., \& Glazounov, A. E. (1999). Does freezing in $\mathrm{PbMg}_{1 / 3} \mathrm{Nb}_{2 / 3} \mathrm{O}_{3}$ relaxor manifest itself in nonlinear dielectric susceptibility? Appl. Phys. Lett., Vol. 74, No. 13, pp. 1910-1912.

Thapliya, R., Okano, Y., \& Nakamura, S. (2003). Electrooptic characteristics of thin-film PLZT waveguide using ridge-type mach-zehnder modulator. J.Lightwave Technol., Vol. 21, No. 8, pp. 1820.

Wei, F., Sun, Y., Chen, D., Xin, G., Ye, Q., Cai, H., \& Qu, R. (2011). Tunable external cavity diode laser with a PLZT electrooptic ceramic deflector. IEEE Photonics Technology Letters, Vol. 23, No. 5, pp. 296. 
Ye, Q., Dong, Z., Fang, Z., \& Qu, R. (2007). Experimental investigation of optical beam deflection based on PLZT electro-optic ceramic. Opt. Express, Vol. 15, No. 25, pp. 16933-16944.

Zhang, Jingwen W., Yingyin K. Zou, Kewen K. Li, Qiushui Chen, Hua Jiang, Xuesheng Chen, and Piling Huang. 2009. Laser action with Nd3 doped electro-optic lead lanthanum zirconate titanate ceramics. Paper presented at Conference on Lasers and Electro-Optics/International Quantum Electronics Conference. 


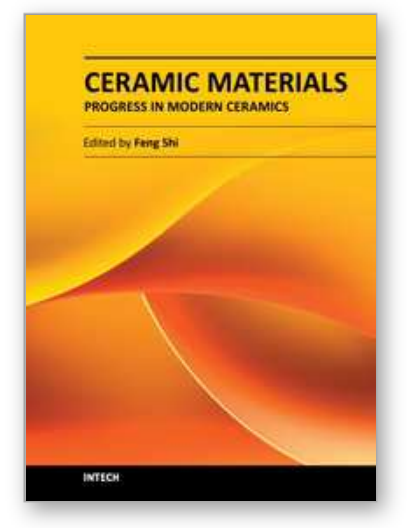

\author{
Ceramic Materials - Progress in Modern Ceramics \\ Edited by Prof. Feng Shi
}

ISBN 978-953-51-0476-6

Hard cover, 228 pages

Publisher InTech

Published online 05, April, 2012

Published in print edition April, 2012

This text covers ceramic materials from the fundamentals to industrial applications. This includes their impact on the modern technologies, including nano-ceramic, ceramic matrix composites, nanostructured ceramic membranes, porous ceramics, and the sintering theory model of modern ceramics.

\title{
How to reference
}

In order to correctly reference this scholarly work, feel free to copy and paste the following:

Ribal Georges Sabat (2012). Characterization of PLZT Ceramics for Optical Sensor and Actuator Devices, Ceramic Materials - Progress in Modern Ceramics, Prof. Feng Shi (Ed.), ISBN: 978-953-51-0476-6, InTech, Available from: http://www.intechopen.com/books/ceramic-materials-progress-in-modernceramics/characterization-of-plzt-ceramics-for-optical-sensor-and-actuator-devices

\section{INTECH}

open science | open minds

\section{InTech Europe}

University Campus STeP Ri

Slavka Krautzeka 83/A

51000 Rijeka, Croatia

Phone: +385 (51) 770447

Fax: +385 (51) 686166

www.intechopen.com

\section{InTech China}

Unit 405, Office Block, Hotel Equatorial Shanghai

No.65, Yan An Road (West), Shanghai, 200040, China

中国上海市延安西路65号上海国际贵都大饭店办公楼405单元

Phone: +86-21-62489820

Fax: $+86-21-62489821$ 
(C) 2012 The Author(s). Licensee IntechOpen. This is an open access article distributed under the terms of the Creative Commons Attribution 3.0 License, which permits unrestricted use, distribution, and reproduction in any medium, provided the original work is properly cited. 